J. Clin. Chem. Clin. Biochem.

Vol. 19, 1981, pp. 1107-1115

\title{
Optimierung der Probenverteilung in einem zentralisierten klinisch-chemischen Institut
}

\author{
Von H. Sator, D. Neumeier und M. Knedel \\ Institut für Klinische Chemie (Direktor: Prof. Dr. med. M. Knedel) Klinikum Großhadern, \\ Ludwig-Maximilians-Universität München
}

(Eingegangen am 23. März 1981)

Zusammenfassung: Mechanisierte, von leistungsfähigen Labordatenverarbeitungssystemen ansteuerbare Proben-Verteilgeräte sind bislang nur in wenigen, zentralisierten klinisch-chemischen Instituten implementiert. Durch eine permanente und direkte, sowohl visuell als auch maschinenlesbare Identifikation der Sekundärgefäße und eine parallele Verteilung auf ausreichend viele Arbeitsplätze werden Identifikationsfehler und Engpässe in der Probenverarbeitung vermieden. Unsere Erfahrungen zeigen aber, daß die Probenverteilung notwendigerweise über diese grundsätzlichen Erfordernisse hinaus weiter zu optimieren ist. Die Verteilung muß insbesondere an die interne Labororganisation und an die Problematik individueller Proben mit in der Analytik interferierenden Substanzen oder mit inadäquatem Probenvolumen angepaßt werden. Neben einer sicheren Probenidentifikation sind die Sekundärgefäße mit dem Probenabnahmedatum und der Materialart zu kennzeichnen, um eine kumulative Abarbeitung seltener Untersuchungen und die simultane Bearbeitung verschiedener Materialien in einer Analysenserie zu ermöglichen. Darüberhinaus ist eine geeignete untersuchungs- oder gerätespezifische Durchführung der internen Qualitätskontrolle zu berücksichtigen. Wir haben diese zusätzlichen Erfordernisse durch eine konsequente gegenseitige Abstimmung im Aufbau der Untersuchungsanträge (Markierung von Prioritäten, Zusatzinformationen), der Dateistrukturen und des Steuerprogramms des Proben-Verteilgerätes erfüllt.

\section{Optimization of sample splitting in a centralized clinical chemical institute}

Summary: Mechanized sample splitting machines controlled by a laboratory data processing system have been realized in only a few centralized laboratories. Bottlenecks and mistakes in sample processing are avoided by means of direct machine readable identification and parallel splitting of the secondary tubes. Our previous experience has shown that a strategy for sample splitting has to go far beyond these basic functional requirements. The splitting process must be suited to the organization of the particular laboratory, and it must be adjusted to deal with problems of individual samples containing analytically interfering substances, or variable splitting may be required in cases of inadequate sample volumes. In addition to sample identification, the secondary tube has to be coded with the date and the type of material. This allows cumulative on-line processing and series of analyses of different materials. A suitable positional arrangement of containers for control material must be born in mind for quality control performances. We have realized these additional requirements by means of a consequent mutual adaptation in the layout of the request form (marking of priority and additional information), the file structure of the data processing system and the control program of the sample splitting machine.

\section{Einführüng}

Zur Vermieidung von Redundanz und Kosten bei der Bestimmung klịnisch-chemișcher Kenngröß̣en ịst bei der Anforderung klinisch-chemischer Untersuchungen eine diskriminierte Auswahl sinnvoll (1-4).

Diese diskriminierte Untersuchungsstrategie führt in einem zentralisierten klinisch-chemischen Institut mit großen Probenzahlen bei der gegenwärtigen Vielzahl von Analysengeräten zu Problemen bezüglich einer sicheren und effizienten Verteilung des Probenmaterials an die entsprechenden Arbeitsplätze (6). Diese Probleme können ohne Unterstützung eines zentralen Labordatenverarbeitungssystems und Implementierung eines teil- oder vollmechanisierten Verteilgerätes nur unbefriedigend gelöst werden.

Die Probenverteilung wird deshalb im Institut für Klinische Chemie am Klinikum Großhadern der Universität München seit 5 Jahren von einem mechanisierten Proben-Verteilgerät (Siemens Typ 4001 VA) vorgenommen. Dieses Gerät, das in Zusammenarbeit mit der Industrie entwickelt wurde, ist an einen Prozeßrechner on-line angeschlossen. Es kann abhängig vom beantragten Un- 
tersuchungsmuster 100-200 Proben pro Stunde verteilen. Dabei werden die Proben in visuell und durch Maschinencodierung permanent und direkt identifizierte Sekundärgefäße dosiert (SILAB-System, Ubersicht bei Knedel (5)) und in bis zu 8 Probenketten für unterschiedliche Analysengeräte ausgeschleust. Zwar wurde die Notwendigkeit einer Mechanisierung in dieser präanalytischen Phase in den vergangenen Jahren in Erwartung neuer diskreter Vielkanal-Analysatoren vereinzelt in Frage gestellt (6), doch gerade in jüngster Zeit wurde das Problem durch die Neuentwicklung mechanisierter Verteilgeräte (7), die Konzipierung von Identifikationssystemen (8) und dem Versuch, die Terminologie des Problemkreises zu vereinheitlichen (9), erneut aufgegriffen.

Obwohl sich das von uns verwendete Proben-Verteilgerät im Routineeinsatz langjährig bewährte und die grundlegenden Erfordernisse einer effizienten Probenverteilung, nämlich hohe Sicherheit und Schnelligkeit, erfüllte, muß nach unseren Erfahrungen der logistische Prozeß zu einer Verteilstrategie weiterentwickelt werden, die es erlaubt, die Verteilung einerseits an die Problematik individueller Proben und andererseits an die jeweiligen organisatorische und apparative Struktur des Labors anzupassen.

Es war deshalb das Ziel bei der Entwicklung des neuen Labordatenverarbeitungssystems unseres Instituts (PRIMULAB) folgende, weitergehende Vorstellungen zur Optimierung der Probenverteilung zu realisieren:

1. Anpassung an die Laborstruktur durch Rechneransteuerung des Proben-Verteilgerätes mit verschiedenen Verteil-Grundprogrammen, um sequentiell die Verteilung von Probenmaterial auf möglichst viele Arbeitsplätze, z.B. Serumchemie I und II, Immunologie und Endokrinologie, zu ermöglichen.

2. Verteilung in Abhängigkeit von probenspezifischen Interferenzen (z. B. hämolytisches Probenmaterial).

3. Verteilung unerwartet kleiner oder kleinster Probenmengen nach definierten Prioritätsfolgen, die auf dem Untersuchungsantrag durch den Antragsteller vorgegeben werden können.

4. Kennzeichnung von Sekundärprobengefäßen $\cdot$ mit der Materialart zur einfacheren materialgerechten Zuordnung von Meßergebnissen durch Analysengeräte im on-line Betrieb.

5. Verteilung des Probenmaterials auf zwei Sekundärgefäße innerhalb einer Analysenkette.

6. Verteilung vorcodierter, leerer Sekundärgefäße zur internen Qualitätskontrolle.
Im folgenden soll gezeigt werden, wie diese Forderungen durch eine konsequente gegenseitige Abstimmung in der Gestaltung der Untersuchungsanträge, in der Systemund Dateikonfiguration der Datenverarbeitungsanlage und des Steuerprogramms des Verteilgerätes realisiert werden können. Am Beispiel des von uns verwendeten Proben-Verteilgerätes wird versucht, wesentliche, allgemein verwendbare Funktionen zu skizzieren.

\section{Optimierung der Probenverteilung}

\subsection{Untersuchungsanträge}

Die von den klinischen Einheiten eingereichten Untersuchungsanträge, die speziell nach den Erfordernissen eines EDV-kompatiblen Informationsträgers konzipiert sind (10), werden im Institut mit einer tagesorientierten, fortlaufenden Probennummer (Tagesnummer) versehen und über eine Lesezange (11) in die Datenverarbeitungsanlage eingelesen. Unter dieser Tagesnummer, die im täglichen Routinebetrieb leichter perzeptibel ist als die siebenstellige Aufnahmenummer des Patienten (Patienten-Aufnahmenummer), wird die gesamte, auf dem Beleg markierte Information in eine Tagesnummer-orientierte Datei gespeichert. Der dabei aufgebaute Datenblock enthält zwingend Patienten-Aufnahmenummer, Einlesedatum, Einleseuhrzeit, Empfangsadresse des Befundes, Belegkennzeichen und fakultativ Informationen zur Abnahme (Bedingungen, Menge, Datum, Zeit), Materialart, Diagnosen, Hinweise auf mögliche medikamentöse Interferenzen, belegorientiertes Eilig-Kennzeichen, zusätzliche Empfangsadresse und ähnliches. Außerdem wird innerhalb dieses Blockes für jede beantragte analytische Untersuchung ein Unterblock aufgebaut, der die Codenummer der Untersuchung, die sog. Verfahrensnummer enthält. Fakultative Einträge sind hier u.a. verfahrensorientiertes Eilig-Kennzeichen und das Kennzeichen ,vorrangig“. Der Begriff ,vorrangig“ bezeichnet eine Kennung, mit der vom beantragenden Arzt, unabhängig von der Dringlichkeit der Ergebnisübermittlung, bestimmte, für den Einzelfall wichtige Untersuchungen markiert werden können, wenn die vorhandene Probenmenge für das gesamte beantragte Untersuchungsmuster unzureichend sein könnte. Durch die Markierung bestimmter analytischer Untersuchungen mit dem Kennzeichen ,vorrangig“" ist es möglich, die Probenverteilung von starren Prioritätsfolgen (z. B. Elektrolyte, harnpflichtige Substanzen, Enzyme usw.) unabhängig zu machen und sie nach einer extern beeinflußten, dem Einzelfall angepaßten Prioritätsfolge zu steuern (s.u.). Mit dem Einlesen des Antrags werden in den Datenblock der Datenverarbeitungsanlage durch Koppelung mit dem ${ }_{0}$ Rechner der Patientenaufnahme die Patientenstammdaten und in jeden verfahrensorientierten Unterblock das - falls vorhanden - jeweils vorausgegangene Ergebnis dieser Untersuchung (Vorwert) mit dem Untersuchungsdatum übernommen. 


\subsection{Rechnerunterstützte, mechanisierte Probenverteilung}

\subsubsection{Kurzbeschreibung des mechanisierten Proben-Verteilgerätes}

\subsubsection{Funktionsablauf (Abb. 1)}

Bauelemente des Proben-Verteilgerätes sind Eingabetastatur, Probenzuführung, Codierstanze, Dosierer und Sortierer. Zur Verteilung wird zunächst das Primärgefäß mit dem Probengut, in der Regel mit Zentrifugationshilfen zentrifugiertes Vollblut, in die Probenzufuihrung der Primärebene eingehängt. Uber eine Eingabetastatur wird die tagesorientierte Probennummer der Datenverarbeitungsanlage mitgeteilt. Da vorher über die Lesezangen die Untersuchungsanträge mit ihrer jeweiligen Patientennummer und Tagesnummer eingelesen werden, kann das ProbenVerteilgerät von der Datenverarbeitungsanlage über ein Ausgabeprogramm entsprechend der beantragten Untersuchungen angesteuert werden. Die notwendige Information ist in einem 15 Byte langen Datensatz (Sekundär-Datensatz, siehe unten) enthalten, der in der Zentraleinheit für jedes Sekundärgefäß aufgebaut und in einem Hardware-Puffer $z$ wischengespeichert wird, bis das Proben-Verteilgerät diese Sätze abruft und weiterverarbeitet. Durch diesen Hardware-Puffer wird ein Blockieren des Ausgabeprogramms in der Datenverarbeitungsanlage vermieden, so daß von diesem Programm ohne nennenswerten Sof tware-Aufwand zwei oder mehrere Proben-Verteilgeräte simultan ohne Zeitverzögerung angesteuert werden können.

Die Probenzuführung des Gerätes übernimmt den Transport des Primärgefäßes zur Dosierposition. Hier wird das Probengut durch den Dosierer in einer von der Datenverarbeitungsanlage festgelegten Menge abgesaugt und entsprechend den jeweils beantragten analytischen Untersuchungen im Bereich der Sekundärebenc auf bereits codierte Sekundärgefäße (Einzeluntersuchungsgefäße) verteilt. Diese Einzeluntersuchungsgefäße werden parallel zum Transfer der Primärprobe durch die Codierstanze codiert. Zur permanenten und direkten Identifikation der Proben werden die Tagesnummer und weitere Informationen (siehe unten) am Fuße der Einzeluntersuchungsgefäße maschinenlesbar gestanzt und am Kopf visuell lesbar geprägt. Die gestanzte Information besteht immer aus 12 Zeichen (11 Zeichen Information, 1 Zeichen zur Parity-Kontrolle), während die Anzahl der visuell lesbaren Stellen bis 11 einstellbar ist. Der weitere Trans-

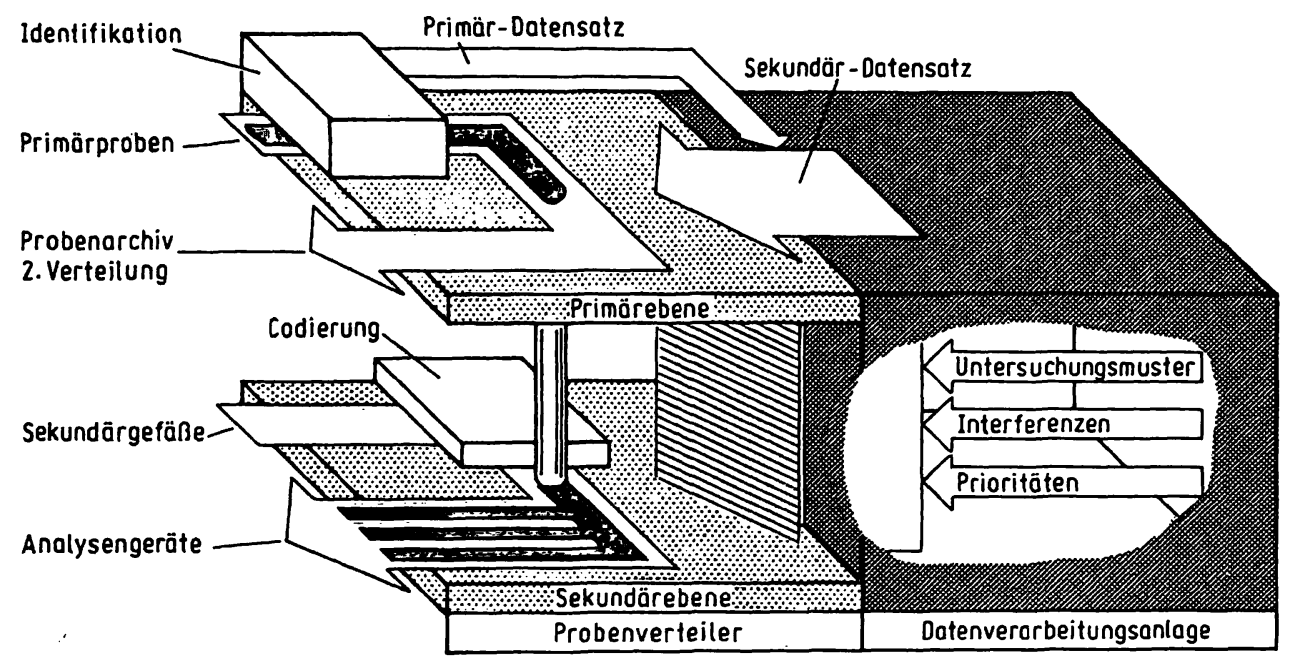

Abb. 1. Proben- und Informationsfluß bei rechnerunterstützter, mechanisierter Probenverteilung mit permanenter, direkter Identifikation der Sekundärproben.

Primär-Datensatz

\begin{tabular}{|c|c|c|c|c|}
\hline 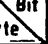 & $2^{3}$ & $2^{2}$ & $2 !$ & $2^{0}$ \\
\hline 0 & \multirow{7}{*}{\multicolumn{4}{|c|}{$\begin{array}{l}\text { Tages-Nummer } \\
\text { oder } \\
\text { Patienten- } \\
\text { Aufnọhmenummer } \\
\text { mit Prüfżiffer }\end{array}$}} \\
\hline 1 & & & & \\
\hline 2 & & & & \\
\hline 3 & & & & \\
\hline 4 & & & & \\
\hline 5 & & & & \\
\hline 6 & & & & \\
\hline 7 & \multicolumn{4}{|c|}{ Interferenzen } \\
\hline 8 & \multirow{3}{*}{\multicolumn{4}{|c|}{ Sonderinfọrmọtiọn }} \\
\hline 9 & & & & \\
\hline 10 & & & & \\
\hline 11 & \multirow{3}{*}{\multicolumn{4}{|c|}{-1}} \\
\hline 12 & & & & \\
\hline 13 & & & & \\
\hline 14 & $\begin{array}{c}1 / 4 \\
\text { volume }\end{array}$ & Volume & & \\
\hline
\end{tabular}

Sekuñnär - Datensatz

\begin{tabular}{|c|c|c|c|}
\hline 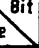 & $2^{3}$ & $2^{1}$ & $2^{0}$ \\
\hline 0 & \multirow{2}{*}{\multicolumn{3}{|c|}{ Tages - Datum }} \\
\hline 1. & & & \\
\hline 2 & \multicolumn{3}{|l|}{ Materialart } \\
\hline 3 & \multirow{3}{*}{\multicolumn{3}{|c|}{ Tages - Nummer }} \\
\hline 4 & & & \\
\hline 5 & & & \\
\hline 6 & & & \\
\hline 7 & \multirow{4}{*}{\multicolumn{3}{|c|}{ Sonderinformọtion }} \\
\hline 8 & & & \\
\hline 9 & & & \\
\hline 10 & & & \\
\hline 11 & \multicolumn{3}{|c|}{ Ausgangs-Kanal Nr. } \\
\hline 12 & \multicolumn{3}{|c|}{ Menge $(\times 150 \mu 1)$} \\
\hline 13 & \multicolumn{3}{|c|}{ Leerverteilung } \\
\hline 14 & Folgebearbeltung & & $\begin{array}{l}\text { Block- } \\
\text { ende }\end{array}$ \\
\hline 15 & & Grundpros & grommintr. \\
\hline
\end{tabular}

Abb. 2. Aufbau der Datenblöcke, die vom Proben-Verteilgerät an die Zentraleinheit (Primär-Datenblock) und von der Zentraleinheit an das Proben-Verteilgerät (Sekundär-Datenblock) abgegeben werden. port der Einzeluntersuchungsgefäße wird durch den Sortierer übernommen. Im ersten Schritt werden sie dem Dosierer zugeführt und mit Probengut befuillt. Im zweiten Schritt werden die Einzeluntersuchungsgefäße über Transportkämme zu den entsprechenden Ausgangskanälen weitergeschoben und in die Probenketten der Analysengeräte ausgesteuert. Die permanent und direkt identifizierten, gefüllten Sekundärgefäße stehen damit zur Untersuchung an den entsprechenden Arbeitsplätzen zur Verfügung. Die weitere Bearbeitung der Primärprobe (erneute Verteilung mit geändertem Grundprogramm, Verwahrung im Probenarchiv) wird über Anzeigefelder auf der Eingabetastatur mitgeteilt. Alle Vorgänge, nämlich Codierung, Dosierung und Verteilung laufen taktverschoben für mehrere Proben parallel ab und erhöhen die Effektivität der Probenverteilung. Während z. B. die Proben $x_{1}, x_{2}, x_{3}$ verteilt werden, wird die Probe $x_{4}$ dosiert und für die Probe $x_{5}$ werden die Einzeluntersuchungsgefäße gestanzt.

\subsubsection{Datenblöcke (Abb. 2)}

Zum Transfer der Informationen, die zur Steuerung des ProbenVerteilgerätes und der permanenten und direkten Identifikation des Sekundär-Probengefäßes notwendig sind, müssen ein Primär-Datenblock und mehrere Sekundär-Datenblöcke aufgebaut werden.

\section{Primärdatenblock}

Die ersten 7 Bytes des 15 Bytes langen Datenblockcs, der von der Eingabetastatur am Proben-Verteilgerät zur Zentraleinheit abge- 
geben wird, dienen der Probenidentifikation. Sie können wahlweise eine dreistellige Tagesnummer oder eine siebenstellige $\mathrm{Pa}$ tientennummer mit Prüfziffer enthalten. Im ,PRIMULAB“-System wird auf der Ebene der Meßwerterfassung ausschließlich mit der Tagesnummer gearbeitet. Dies hat neben der besseren Perzeptibilität den Vorteil, daß 4 Bytes zur Verfügung stehen, die für spezifische Steuerfunktionen (Abnahme-Datum, Materialart) genutzt werden können. Um die Probenverteilung an die Organisationsstruktur des Labors, definierte Probencharakteristika und an das aktuell zur Verfügung stehende Probenvolumen anzupassen, werden 3 weitere Bytes für entsprechende Sonderinformationen, die am Proben-Verteilgerät mit der Tagesnum mer über die Tastatur eingegeben werden, in den Primärdatensatz aufgenommen.

\section{Sekundärdatenblock}

Durch das. Eintreffen des Primär-Datenblocks in der Datenverarbeitungsanlage wird mit Hilfe der Information dieses Blockes und der des entsprechenden Untersuchungsantrages für jedes einzelne Sekundärgefäß im on-line Betrieb ein Sekundär-Datenblock aufgebaut, der 16 Bytes lang ist und an das Próben-Verteilgerät abgegeben wird. In diesen Datenblock wird die Kennung für das Annahmedatum der Probe und die Materialart aufgenommen. Die Information zum kanalabhängigen Ausschleusen von leeren, vorcodierten Sekundärgefäßen für Qualitätskontrollproben sowie zur weiteren Bearbeitung der Primärprobe (Signalfeld der Eingabetastatur) wird über Byte 13 und 14 an das Proben-Verteilgerät übergeben. Der jeweils letzte Sekundär-Datenblock, der von einem Primärgefäß (bzw. einer Tagesnummer) induziert wird, wird ebenfalls im Byte 14 gekennzeichnet.

\subsubsection{Anpassung an die Organisationsstruktur des Labors}

Ein Ziel effizienter Probenverteilung ist es, zur Verkürzung der Abarbeitungszeit vom Eintreffen des Untersuchungsmaterials bis zur Ausgabe des Befundberichtes beizutragen. Dieser Aufgabe wird bevorzugt ein paralleles Aufteilen der Primärprobe in Sekundärproben gerecht. Für mechanisierte Systeme ist ein paralleles, gleichzeitiges Ausschleusen der Sekundärprobengefäße auf möglichst viele Analysenketten der Sekundärebene anzustreben. Allerdings ist von technischer Seite die Zahl der Ausgangs-Kanäle begrenzt. Aus diesem Grund sowie zur Ausfallsicherung werden von uns zwei Proben-Verteilgeräte eingesetzt. Diese Geräte können parallel mit einem Verteilprogramm oder aber, da vom Antragsteller bereits eine Vorverteilung des Probenmaterials auf einzelne Untersuchungsgefäße für immunologische oder endokrinologische Untersuchungen vorgenommen wird, mit verschiedenen Verteilprogrammen angesteuert werden. Die Wahl des jeweiligen Grundprogramms, das die Verteilung fur die Bereiche Serumchemie I oder II, Immunologie oder Endokrinologie steuert, erfolgt durch einmalige Tastatur-Eingabe der entsprechenden Programmnummer als Sonderinformation im Primär-Datensatz (Bytes 8-10) mit der ersten Tagesnummer. Das gewählte Grundprogramm des Proben-Verteilgerätes wird auf einem Anzeigefeld der Eingabetastatur angezeigt. Für jedes dieser Verteil-Grundprogramme existiert in der Datenverarbeitungsanlage im Probenverteilprogramm (,PRVERT“) eine Liste (Abb. 3), die zu jedem Kanal zwei Mengenangaben (,Menge I“ und „Menge Iİ“) und eine Verfahrensnummern-Unterliste enthält. Das Spektrum der analytischen Untersuchungen (Verfahrens-

\begin{tabular}{|c|c|c|c|}
\hline \multicolumn{4}{|c|}{ Grundprogramm Nr. } \\
\hline \multirow{3}{*}{$\begin{array}{c}\text { Ausgongs-Konal } \\
\text { Nr. } 1\end{array}$} & \multirow{3}{*}{ Menge I } & \multirow{3}{*}{ Menge II } & Verfahrens-Nummer \\
\hline & & & $\quad \quad \vdots$ \\
\hline & & & Verfohrens-Nummer \\
\hline \\
\hline \multirow{3}{*}{$\begin{array}{c}\text { Ausgangs-Kanal } \\
\text { Nr. } 8\end{array}$} & \multirow{3}{*}{ Menge I } & \multirow{3}{*}{ Menge II } & Verfahrens-Nummer \\
\hline & & & $=\quad \vdots$ \\
\hline & & & Verfahrens - Nummer \\
\hline
\end{tabular}

Abb. 3. Schematischer Aufbau einer Verteilliste im Steuerprogramm des Proben-Verteilgerätes.

nummern-Unterliste) pro Kanal hängt von der Zahl der vom Analysengerät parallel durchgeführten Untersuchungen bzw. dem Arbeitsplatz ab; an dem die entsprechende Probenkette abgearbeitet wird. Entsprechend der aktuellen Erfordernisse kann dieses Spektrum bei Totaloder Teilausfall eines Analysengerätes kurzfristig geändert werden. Dem Proben-Verteilgerät ist während des Verteilvorgangs eine dieser Listen zugeordnet. Für jeden Kanal in dieser Liste wird nach Prüfung, ob mindestens eine dem Kanal zugeordnete analytische Untersuchung unter der eingegebenen Tagesnummer beantragt ist, ggf. ein Sekundär-Datenblock mit der in der Liste angegebenen ,Menge I" aufgebaut und dem Verteilgerät übergeben. Bei einem Ausbau des Verteilgerätes mit 8 Kanälen zum Ausschleusen der Sekundärproben werden 1-8 Datensätze pro Tagesnummer erzeugt, wobei der letzte durch das Ende-Bit gekennzeichnet sein muß. Falls keine Anforderung für einen der Kanäle existiert, muß ein leerer Pseudo-Datensatz mit EndeKennzeichen erzeugt werden.

\subsubsection{Verteilen in Abhängigkeit von probenspezifischen Interferenzen}

Die Richtigkeit der klinisch chemischen Untersuchungsergebnisse wird wesentlich bestimmt durch das frühzeitige Erkennen und Eliminieren probenspezifischer Störgrößen, die bei der Analytik interferieren. Während der Probenverteilung ist es deshalb wichtig, Interferenzen durch hämolytische, ikterische oder lipämische Seren zu erkennen und von der Verteilung auf hiervon beeinflußte analytische Untersuchungen auszuschließen. Daneben ist diese Information sofort an die den Patienten betreuende Einheit mitzuteilen, um dort frühzeitig eine Reaktion (z.B. neue Probennahme) auslösen zu können. Vom ,PRIMULAB-System“ können die Charakteristika „hämolytisch“, ,lipämisch“ und ,ikterisch“ unabhängig voneinander berücksichtigt werden. Entsprechende Kennungen werden als Steuerinformation am Verteilgerät über Tastatureingabe in den Primär-Datensatz (Byte 7) aufgenommen. In der Verfahrensdatei der Datenverarbeitungsanlage ist für jede Untersuchung angegeben, ob die analytische Durchführing bei den jeweiligen Interferenzen methodisch unmöglich bzw. bedingt möglich 
ist. Entsprechend werden für die weitere Verarbeitung bis zu drei Alternativen unterschieden. Dies sei am Beispiel einer hämolytischen Probe erläutert (Abb. 4).

1) Die beantragte Untersuchung wird über das ProbenVerteilgerät verteilt, methodisch wird das Ergebnis durch Hämolyse beeinflußt.

In diesem Fall wird als Untersuchungsergebnis der Qualitativ-Wert „HAEM“ übergeben, der später als Text auf den Befundberichten erscheint. Trifft dieser Fall auf alle Untersuchungen eines Ausgangs-Kanals zu, so unterläßt das Programm eine Verteilung des Probenmaterials auf diesen Kanal.

2) Die beantragte Untersuchung wird über das ProbenVerteilgerät verteilt, methodisch wird das Ergebnis durch Hämolyse nicht beeinflußt.

In diesem Fall wird dem später eintreffenden Ergebnis das Kennzeichen „H“ für hämolytisch beigefügt. Auch in den Sonderfällen, in denen die auf dem Untersuchungsantrag markierten Zusatz-Informationen zeigen, daß eine Hämolyse zum Zeitpunkt der Probengewinnung unvermeidbar ist, $\mathbf{z}$.B. während extrakorporaler Zirkulation, wird ein durch Hämolyse beeinflußtes Untersuchungsergebnis (z.B. Kalium) mit diesem Vermerk versehen.

3) Die beantragte Untersuchung wird nicht über das Proben-Verteilgerät verteilt.

In diesem Fall wird zunächst als „Ergebnis“ der Wert „HAEM“ eingetragen, der in Abhängigkeit von den Methodenkriterien in der Verfahrensdatei ggf. durch ein quantitatives oder qualitatives Ergebnis mit der Zusatzinformation „H“ überschrieben werden kann.

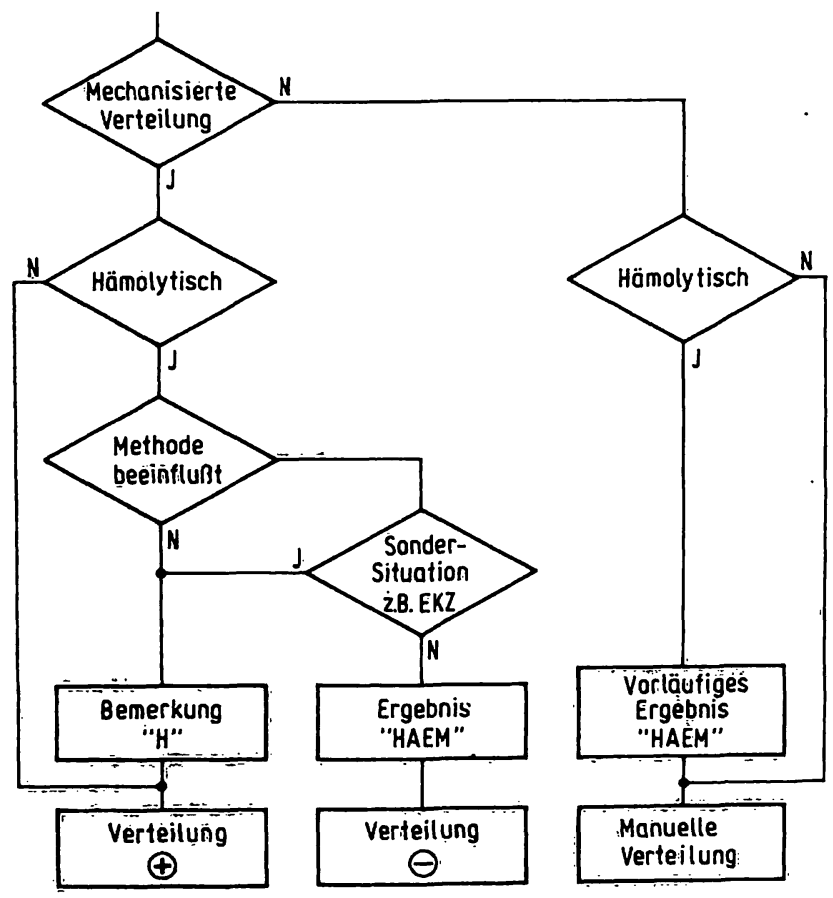

Abb. 4. Schema dẹ Probenverteilung bei hämolytischen Serumproben.
In gleicher Weise werden bei einem Mehrkanal-Analysator, der simultan sowohl durch Hämolyse beeinflußte als auch nicht beeinflußte Untersuchungen durchführt, hämolysebedingte falsche Ergebnisse unterdrückt, wenn bereits ein vom Probenverteilprogramm eingetragener Qualitativwert „HAEM“ vorliegt. Daneben steuert das Programm „PRVERT“ den sofortigen Ausdruck einer entsprechenden Mitteilung an die beantragende Einheit.

\subsubsection{Verteilung in Abhängigkeit vom aktuellen Probenvolumen}

Ein häufiges Entscheidungsproblem klinisch chemischer Zentrallaboratorien entsteht aus einem Mißverhältnis von verfügbarem Probenvolumen und der $Z$ ahl der beantragten analytischen Untersuchungen (Abb. 5). In diesen Fällen müssen für die Verteilung zusätzliche, möglichst externe (siehe oben) Informationen beim Festlegen der Prioritätsfolge berücksichtigt werden. Die Informationen, daß nur ,wenig" oder ,sehr wenig“" Probenmaterial zur Verfügung steht, wird als Steuerinformation durch Tastatureingabe im Primär-Datensatz (Byte 14) der Zentraleinheit mitgeteilt. Ohne Kenntnis der Zahl der beantragten analytischen Untersuchungen wird bei der Eingabe das Volumenmerkmal "wenig" bzw. „sehr wenig“ gewählt, wenn das primäre Probengefäß $(20 \mathrm{ml})$ nur halb bzw. bis zu einem Viertel gefüllt ist.

\section{Volumenmerkmal ,wenig“}

Nach Vergleich dieser Volumeneingabe mit dem beantragten Untersuchungsmuster wird durch das Steuerprogramm der Sekundär-Datenblock aufgebaut, wobei gegebenenfalls statt der ,Menge I“" die „Menge II“ eingetragen wird. Im Gegensatz zur „Menge I“, die ein Reservevolumen beinhaltet, das die Wiederholung der Untersuchung am Analysengerät bei gestörter Präzisions-

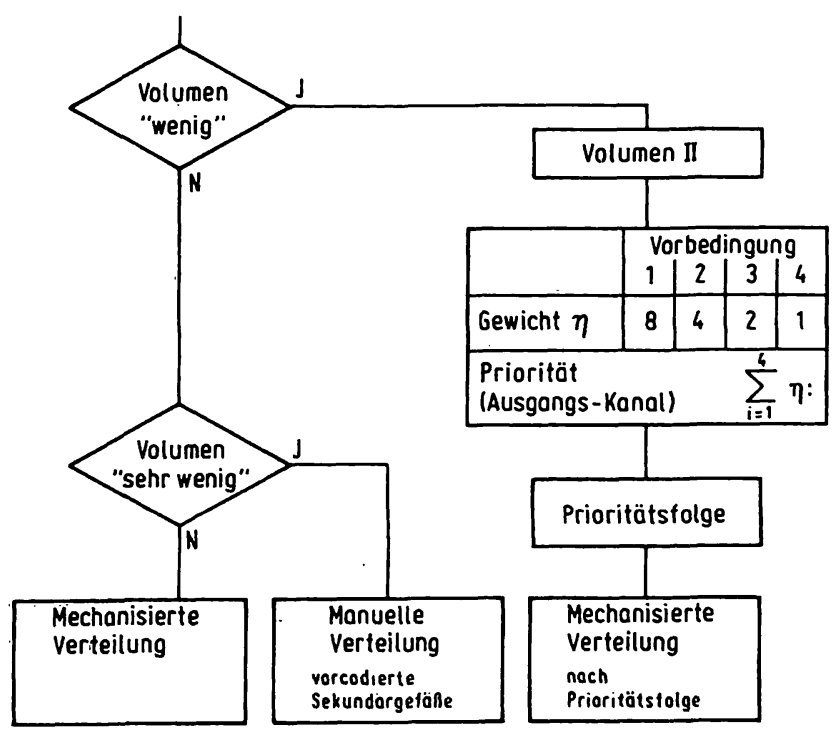

Abb. 5. Schema der Probenverteilung bei inadäquatem Probenvolumen. Aufbau der Prioritätsfolge. 
oder Richtigkeitskontrolle zuläßt, wird im Modus „Menge II" das Mindestvolumen verteilt, das vom Analysengerät benötigt wird, um aus der Probe einen gültigen Meßwert zu erstellen. Darüberhinaus erfolgt die Verteilung nach einer Prioritätsfolge, die entsprechend definierter Vorbedingungen aufgestellt wird. Nach ihrer Gewichtung seien diese Vorbedingungen ,PRIO 1" bis „PRIO 4“ genannt, „PRIO X“" ist nur dann von Bedeutung, wenn aufgrund höher gewichteter Vorbedingungen keine Entscheidung getroffen werden kann.

PRIO 1: Die analytische Untersuchung ist auf dem Antrag mit dem Vermerk ,vorrangig" versehen. Damit wird die oben beschriebene externe Einflußnahme auf die Probenverteilung ermöglicht.

PRIO 2: Das vorausgehende Untersuchungsergebnis ist pathologisch, d.h. es liegt außerhalb'des Referenzbereiches.

PRIO 3: Verteilung in Abhängigkeit vom Zeitintervall zum Vorwert.

PRIO 4: Verteilung nach einer im Steuerprogramm vorgegebenen Prioritätsfolge (z.B. Elektrolyte, harnpflichtige Substanzen, Enzyme usw.).

\section{Volumenmerkmal „sehr wenig“}

Nach Vergleich mit dem beantragten Untersuchungsmuster wird die Probe gegebenenfalls nicht über das Proben-Verteilgerät verteilt. Es werden entsprechend dem Untersuchungsmuster Einzeluntersuchungsgefäße gestanzt, auf die die Primärprobe nach Rückfrage bei dem beantragenden Arzt von Hand verteilt wird.

\subsubsection{Sonderinformationen}

Da im „PRIMULAB“-System auf der Ebene der Meßwerterfassung die Probenidentifikation allein über das Symbol der Tagesnummer erfolgt, können die gegenüber der vollständigen Patientennummer freigewordenen Zeichen auf dem Einzeluntersuchungsgefäß zur visuell- und maschinenlesbaren Kennung des Annahmedatums und der Materialart genutzt werden. Diese ersten sechs gestanzten Bytes werden von allen Analysengeräten mit integrierter, direkter Probenidentifizierung im Rohmeßwertsatz der Datenverarbeitungsanlage übergeben. Durch die Identifikation der Einzeluntersuchungsgefäße mit dem Datum wird es möglich, Untersuchungsanträge und Probenmaterial für Untersuchungen, die nicht täglich on-line abgearbeitet werden (z.B. quantitative Immunglobulinbestimmung), zu kumulieren und bei Abarbeitung den Meßwert der bis zu 28 Tage zurückliegenden Probenannahme automatisch zuzuordnen.

Durch die Kennung des Sekundärgefäßes mit der Materialart kann bei Untersuchungen, bei denen die Wahl der analytischen Methode nicht durch das Material beeinflußt wird, das Meßergebnis direkt der dem Material entsprechenden Verfahrensnummer zugeordnet werden.
Damit gelingt die Unterscheidung von Sërum und Urin oder dem Probenpaar Serum/Urin (z.B. Clearance-Untersuchungen) innerhalb einer on-line laufenden Analysenserie, die bislang nur über Steuersignale möglich war. Für Clearance-Untersuchungen kann Serum und Urin unter identischer Probennummer paarweise analysiert werden, wobei die Filtrationsrate unter Einbeziehung der auf dem Untersuchungsantrag markierten Sammelzeit und Sammelmenge direkt von der Datenverarbeitungsanlage berechnet und abgelegt wird.

\subsubsection{Verteilung einer Primärprobe auf zwei Sekundärgefäße innerhalb einer Proben- kette}

Das Verteilen der Primärprobe auf zwei Sekundärgefäße (Einzeluntersuchungsgefäße) innerhalb einer Probenkette ist zur Durchführung von Doppelbestimmungen, für Qualitätskontrollzwecke und bei kleinen Sekundärgefäßen zur Verdoppelung der verfügbaren Probenmenge an diskreten Vielkanal-Analysatoren erforderlich. Es ist ein Dosieren auf žwei Sekundärgefäße mit gleicher bzw. unterschiedlicher Tagesnummer möglich.

\section{Verteilung der Primärprobe auf Sekundärgefäße mit gleicher Tagesnummer}

Zur Durchführung von Doppelbestimmungen muß eine Verteilung auf zwei gleich identifizierte Sekundärgefäße möglich sein. Diese Form der Verteilung wird durch eine entsprechende Kennung in der Verfahrensdatei (z.B. on-line abzuarbeitende, homogene Enzym-Immunoassays) verfahrensabhängig gesteuert. Die Sekundärgefäße werden paarweise in die Analysenketten ausgeschleust. Aus dem vom Analysengerät sequentiell mit gleicher Identifikation abgegebenen Rohmeßwertsätzen werden in der Datenverarbeitungsanlage die Mittelwerte gebildet.

\section{Verteilung der Primärprobe auf Sekundärgefäße mit unterschiedlicher Tagesnummer \\ Qualitätskontrolle}

Eine effiziente Form der Präzisionskontrolle und der Erkennung grober Fehler beruht darauf, daß für einzelne, zufällig ausgewählte und an den Arbeitsplätzen unbekannte Patientenproben Doppelbestimmungen durchgeführt und die Abweichungen der Einzelergebnisse protokolliert werden (12). Entsprechende Doppelverteilungen können durchgeführt werden, wenn vor Eingang der Primärproben einzelne Tagesnummern ausgewählt werden, deren Untersuchungsmuster bei ausreichender Pro= benmenge zunächst in gewohnter Weise und dann gewissermaßen als Duplikat auf weitere Sëkundärgefäße verteilt werden soll. Diese Einzeluntersuchungsgefäße werden mit der nachfolgenden Tagesnummer codiert. Üm den Charakter einer unbekannten Probe zu wahren, werden diesen intern vorbesetzten Tagesnummern in den Arbeits- und Ergebnislisten die gespeicherten Personendaten von „Pseudopatienten“ zugespielt. 


\section{Verdoppelung der Probenmenge}

Im Rahmen der Implementierung diskreter VielkanalAnalysatoren in bestehende Organisationsformen kann infolge der relativ kleinen Volumina der Einzeluntersuchungsgefäße das Problem auftreten, daß in Abhängigkeit von Anzahl und Art der beantragten Untersuchungen die für das Meßgerät erforderliche Probenmenge nicht dosiert werden kann. Diese Schwierigkeit ist zu umgehen, wenn von der Datenverarbeitungsanlage bereits während des Einlesens geprüft wird, ob für die über den Vielkanal-Analysator abzuarbeitenden Untersuchungen die maximale Füllmenge eines Einzeluntersuchungsgefäßes ausreichen wird. Ist dies nicht der Fall, so werden dem Antrag intern zwei aufeinander folgende Tagesnummern zugeteilt. Die Verteilung einer Primärprobe auf zwei Sekundärgefäße mit unterschiedlicher Tagesnummer innerhalb einer Analysenkette setzt voraus, daß die fälschliche Doppelvergabe einer Tagesnummer sicher ausgeschlossen ist. Dies ist über einen Tagesnummer-Stempel mit integriertem Belegleser zu erreichen, der von der Datenverarbeitungsanlage zur Vergabe der nächsten freien Tagesnummer angesteuert wird (13). Die korrekte Abarbeitung des auf zwei Tagesnummern verteilten Untersuchungsmusters ist durch einen bidirektionalen on-line Anschluß des Analysengerätes an die Datenverarbeitungsanlage zu steuern. Dieser Anschluß ist zwingende Voraussetzung für einen sinnvollen Einsatz dieser Geräte in einem Zentrallabor, das mit Unterstützung eines Labordatenverarbeitungssystems arbeitet.

\subsubsection{Verteilung vorcodierter, leerer Sekundärgefäße für interne Qualitätskontrollproben}

Im Rahmen der internen Qualitätskontrolle ist die Sequenz der Kontrollproben innerhalb der Probenketten nach Methoden oder gerätespezifischen Kriterien in der Verfahrensdatei festgelegt. Das Proben-Verteilgerät wird entsprechend dieser vorgegebenen Sequenz angesteuert.

\section{Diskussion}

Die șichere und schnelle Verteilung der in Sekundärproben aufgeteilten Primärproben auf einzelne analytische Arbeitsplätze ist wesentliche Voraussetzung für eine funktionelle Bewältigung des täglichen Probeneingangs in zentralisierten klinisch-chemischen Instituten. Neben der Auf- und Verteilung von Probenmaterial ist zu dessèn Identifikation ein vielfältiger Informationstransfer notwendig. Diese Informationsübertragung erfolgt entweder in vollständiger Form, die durch individuelle Personaldaten und Probengewinnung festgelegt ist oder in Form eines Informationssymbols (Tagesnummer), das jederzeit den Zugang zur vollständigen Information erlauben muß. Bei einer manuellen Verteilung steigt die Fehlerrate im Informationstransfer in Abhängigkeit von der Zahl der beantragten Untersuchungen und der in den Verteilprozeß einbezogenen Personen. Nach Untersuchungen von Cole (14) liegt diese Fehlerhäufigkeit pro Person und tausend beantragte Untersuchungen zwischen 6,7 und 118,8 Übertragungsfehlern, je nachdem, ob pro Untersuchungsformular eine oder 5-10 Untersuchungen beantragt werden. Neben dieser Fehleranfälligkeit veranlaßten Kapazitätsprobleme der manuellen Verteilung, die mit dem diskontinuierlichen Eintreffen des Probenmaterials verbunden sind, zur Entwicklung einer Alternative.

Eine Möglichkeit besteht darin, eine Probenverteilung zu umgehen, indem alle Primärproben möglichst einem Vierkanal-Analysator mit fixiertem oder selektivem Untersuchungsprofil zugeführt werden. Die Probenverteilung wird zum geräteinternen Organisationsproblem. Diese geräteinterne Aufteilung wird aber auch künftig, wenn diskrete Vielkanal-Analysatoren in den Labors implementiert sind und mit einem Labordatenverarbeitungssystem bidirektional Daten austauschen, eine Probenverteilung nicht vollständig ersetzen. Vorwiegend aus methodischen Gründen wird weiterhin eine Verteilung auf einzelne restliche Arbeitsplätze (z. B. SerumElektrophorese, definierte Elektrolyte und Spurenelemente, Osmolalität), für die eine ausreichende Serienlänge als ökonomische Grundlage einer apparativen Verteilung vorliegt, notwendig sein.

Als weitere Möglichkeit bietet sich an, die Probenverteilung über ein Labordatenverarbeitungssystem zu steuern und möglichst weitgehend zu mechanisieren.

\section{Mechanisierte Verteilung und Identifikation der Proben}

Ein mechanisiertes Proben-Verteilgerät hat grundlegende Erfordernisse, d.h. höchste Sicherheit vor Probenverwechslungen und -verfälschungen, sowie hohe Schnelligkeit zu erfüllen. Diesen Kriterien werden heute installierte oder konzipierte, mechanisierte Systeme der Probenverteilung (Übersicht bei Helb (7), 15-17) in sehr verschiedener Weise gerecht. Während nach dem Stande der Technik eine Verfälschung des analytischen Ergebnisses durch Verschleppungseffekte weitgehend auszuschließen ist, ist ein verwechslungsfreier Transfer der Identifikation des Primärprobengefäßes auf die Sekundärprobengefäße bislang noch nicht absolut sicher gewährleistet. Nach unserer Erfahrung sind zur sicheren Identifikation Systeme vorzuziehen, die für den Bereich der Sekundärebene eine permanente und direkte Identifikation des Sekundärprobengefäßes ermöglichen. Gegenüber einer Probenidentifikation mit Hilfe einer platzcodierten Zuordnung der Sekundärgefäße bietet die visuelle und maschinenlesbare Codierung der Sekundärprobe den höchstmöglichen Schutz vor Verwechslungen (18). Dies ist insbesondere in zentralisierten Instituten wichtig, die weitgehend auf eine untersuchungsorientierte Vorverteilung des Probenmaterials durch den Einsender verzichten und demzufolge auf zahlreiche, durch mehrere Mitarbeiter ausgeführte Transfervorgänge angewiesen sind. Bei diesen Systemen ergeben sich die Fehler im Transfer der Identifikation vorwiegend auf der Primärebene der Probenverteilung. Bei dem beschrie- 
benen Verteilprozeß finden sich die gegenwärtig störanfälligsten Úbertragungsstellen bei der Vergabe der Tagesnummer und am Verteilgerät bei der visuellen Identifikation der Primärprobe mit folgender Tastatureingabe der Tagesnummer. Eine Lösung dieses Problems ist nur mit relativ hohem technischen Aufwand, beispielsweise durch die Integration eines rechnergesteuerten Tagesnummer-Stempels in ein Proben-Verteilgerät zu erwarten. Wird darauf verzichtet, auch die Primärprobe mit einem leicht perzeptiblen Identifikationssymbol (Tagesnummer) zu markieren, so sind kostengünstigere Lösungen mit einer direkten Lesung der Primärprobenidentifikation zu realisieren.

Unter den verschiedenen, kürzlich zusammenfassend dargestellten Formen des Verteilprozesses (9) ist nach unserer Meinung in zentralisierten Instituten mit ihrer vielfältigen apparativen Ausstattung ein quasi-paralleles Erstellen arbeitsplatzorientierter Probenfolgen vorteilhaft in den gesamten Funktionsablauf zu integrieren. Bei dieser Verteilform ist gewährleistet, daß an mehreren Arbeitsplätzen gleichzeitig mit der Analytik begonnen werden kann. Ein Warten auf das Eintreffen aller zur Verteilung anstehenden Proben ist nicht notwendig. Für Untersuchungen, die für diagnostische und therapeutische Entscheidungen wesentlich sind, können die Proben in der Reihenfolge des Eingangs schnell abgearbeitet werden. Die direkte Identifikation erlaubt darüberhinaus, diese Reihenfolge im Einzelfall an aktuelle Bedürfnisse anzupassen. In unserem Institut erfaßt das durch die apparative Ausstattung beeinflußte Verteilschema auf 8 parallele Ausgangskanäle (Serumchemie I) etwa $80 \%$ der klinisch-chemischen Routine-Untersuchungen. Apparative und ökonomische Grenzen stehen aber einer parallelen Verteilung auf alle Arbeitsplätze oder alle möglichen Untersuchungen entgegen. Das Ausschleusen auf weitere Arbeitsplätze würde neben dem apparativen Aufwand die erforderliche Verteilzeit beträchtlich erhöhen. Es ist günstiger, Proben mit einem über diese $8 \mathrm{Ka}$ näle hinausgehenden Untersuchungsmuster anzuzeigen und mit einem zweiten Grundprogramm auf weitere Ausgangskanäle zu verteilen. Die parallele Probenverteilung in permanent und direkt identifizierte Sekundärgefäße führt aus technischen Gründen zu einer Beschränkung auf einen definierten Gefäßtyp für die Sekundärprobe. Für alle on-line anzuschließenden Analysengeräte muß eine Adaptierung des geräteeigenen Probennehmers an diesen Gefäßtyp vorgesehen werden.

\section{Steuerung der Probenverteilung}

\section{über ein Labordatenverarbeitungssystem}

Zentrales Element einer mechanisierten Probenverteilung ist nach unseren Erfahrungen neben dem Verteilgerät die Steuerung dieses Gerätes über ein leistungsfähiges Labordatenverarbeitungssystem. Ein off-line arbeitendes Proben-Verteilgerät wird die Verteilung im Grunde nur an den manuellen Prozeß angleichen. Es kann dabei die Verwechslungsgefahr reduziert, die Geschwindigkeit erhöht und die dosierte Probenmenge an das Analysenvolumen angepaßt werden. Diese grundlegenden Erfordernisse sind aber auch ohne mechanisches Verteilgerät, allein durch eine rechnergestützte und über Signallampen gesteuerte, manuelle Probenverteilung, kostensparend zu erfüllen (19). Erst der bidirektionale Anschluß an ein zentrales Labordatenverarbeitungssystem rechtfertigt den hohen finanziellen Aufwand für mechanisierte ProbenVerteilgeräte. Dieser Anschluß erlaubt, Verteilstragegien zu entwickeln, die bei gegenseitiger Abstimmung von Untersuchungsanträgen, Dateistrukturen, Steuerprogramm und Verteilfunktionen auf die Problematik individueller Proben ausgerichtet, bei manueller Verteilung aber nicht oder nur bedingt einzuhalten sind. Verteilstrategien, d. h. die Auswahl geeigneter auf Wissen und Erfahrung beruhender Aktionsfolgen, sind insbesondere in zentralisierten Instituten mit großem Probeneingang erforderlich. Hier ist es aus zeitlichen und personellen Gründen unmöglich, bei Proben mit inadäquatem Probenvolumen die Prioritätsfolge der Untersuchungen jeweils durch telefonische Rückfrage festzulegen. Eine starre Prioritätsfolge der Probenverteilung führt nach unseren Erfahrungen zu unbefriedigenden Ergebnissen. Durch die Möglichkeit einer externen Markierung wird zunächst in den Fällen, in denen bereits bei der Probennahme ein Volumen-Mißverhältnis vermutet wird, die gewünschte Prioritätsfolge festgelegt. Der bidirektionale Anschluß des Verteilgerätes an ein Labordatenverarbeitungșsystem ermöglicht darüberhinaus, auch Proben, bei denen erst während der Verteilung ein möglicherweise unzureichendes Probenvolumen signalisiert wird, nach Prüfung in der Datenverarbeitungsanlage in einer nach Vorbedingungen festgelegten, individuellen Prioritätsfolge zu verteilen. Auch die Probleme beim Verteilen von Proben mit chromogenen Interferenzen, die bei manueller Verteilung auch bei gut ausgebildetem Personal nur sehr bedingt und nach häufig nicht festgelegten Entscheidungsfolgen gelöst werden, sind über eine Ansteuerung des Proben-Verteilgerätes in definierter Form zu lösen. Auf bekannte Sondersituationen kann angepaßt reagiert werden.

Im Rahmen der Qualitätskontrolle wird durch die Ansteuerung des Probenverteilers das positionsgerechte Ausschleusen vorcodierter Sekundärgefäße in die Probenketten methoden- und gerätespezifisch kontrolliert. Darüberhinaus ist für Doppelproben, die bei parallel verteilenden Geräten paarweise ausgeschleust werden, ụber gespeicherte Pseudo-Patientendaten der Charakter einer unbekannten Probe gewahrt.

Abschließend ist festzustellen, daß im Bereich zentralisierter Institute durch eine mechanisierte Probenverteilung auf permanent und direkt identifizierte Sekundärgefäße ein hohes Maß an Sicherheit vor Probenverwechslungen erreicht wird. Bei verschiedenen, gleichzeitig arbeitenden Analysengeräten ist eine parallele Verteilung der Primärprobe anzustreben. Die Möglichkeiten der mechanisierten Probenverteilung können aber exst durch die 
Steuerung über ein Labordatenverarbeitungssystem vollständig genutzt werden. Neben der Implementierung vorgegebener Verteilfolgen zur internen Qualitätskontrolle kann die Verteilung vor allem an die Problematik einzelner Proben angepaßt werden. Zukünftige Entwicklungen und Ziele werden sich auf das Ausschalten stör-

\section{Literatur}

1. Keller, H. (1978) J. Clin. Chem. Clin. Biochem. 16, 687692.

2. Sandler, G. (1979) Br. Med. J. 2, 21-24.

3. Haeckel, R. (1980) Die Problematik der ungezielten Mehrfachanalyse - Aus der Sicht des Klinischen Chemikers. In „Validität klinisch-chemischer Befunde" (Lang, H., Rick, W. \& Büttner, H., eds.), Springer-Verlag, Berlin-HeidelbergNew York.

4. Gross, R. \& Oette, K. (1980) Die Problematik der ungezielten Mehrfachanalyse - Aus der Sicht des Klinikers. In „Validität klinisch-chemischer Befunde" (Lang, H., Rick, W. \& Büttner, H., eds.), Springer-Verlag, Berlin-HeidelbergNew York.

5. Knedel, M. (1971) Datenverarbeitung im Klinisch-Chemischen Institut am Städtischen Krankenhaus München-Harlaching - Ein System im Aufbau. Siemens Druckschrift Nr. MC 50/1016.

6. Haeckel, R. (1980) J. Clin. Chem. Clin. Biochem. 18, 455459.

7. Helb, H.-D. \& Assmann, G. (1980) GIT-Labor-Medizin 6, 465-475.

8. Helb, H.-D. \& Assmann, G. (1979) GIT-Labor-Medizin 2, 295-305.

9. Mieth, I. (1980) GIT-Labor-Medizin 6, 457-464. anfälliger Übertragungsstellen im Informationstransfer und eine Weiterentwicklung der Verteilstrategie konzentrieren. Die probenspezifische Anpassung ist durch die Berücksichtigung der klinischen Fragestellung und möglicher medikamentöser Interferenzen weiter $z u$ verbessern.

10. Knedel, M. (1979) Design of a laboratory request sheet for the Institute of Clinical Chemistry of the L.-M. University in Munich, 3rd European Congress of Clinical Chemistry, Brighton.

11. Killian, K. (1976) Verarbeitung von klinisch-chemischen Meßwerten in redundanten Systemen durch externe und interne Variation von Programmstruktur und Meßvorgang. In "Interaktive Datenverarbeitung in der Medizin" (Wagner, G. \& Köhler, C. O., eds.), F. K. Schattauer-Verlag, StuttgartNew York, p. 178-187.

12. Boroviczeny, K. G. von \& Merten, R. (1972) Ärztl. Lab. 18, 26-31.

13. Engelhardt, H.-J. \& Spiegel, K. (1980) The PRIMULAB printing mark sense card readers, "Computing in Clinical Laboratories", Third In ternational Conference, Birmingham.

14. Cole, G. W. (1980) Hum. Pathol. 11, 424-434.

15. Dudeck, J., Roka, L. \& Michel, H. (1974) Ärztl. Lab. 20, 430-436.

16. Froreich, A. von (1978) GIT-Labor-Medizin 3, 197-200.

17. Conradi, R. (1980) Labor-Praxis Med. 11, 67-69.

18. Keller, H. (1979) J. Clin. Chem. Clin. Biochem. 17, 57-64.

19. Froreich, A. von (1980) GIT-Labor-Medizin 3, 37-40.
Dipl. Inf. H. Sator Institut für Klinische Chemie Klinikum Großhadern Postfach 701260 D-8000 München 70 
$$
\int
$$
$\Gamma$

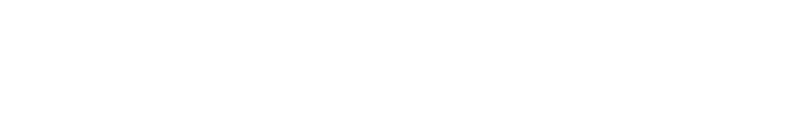

\section{.}

